\title{
De la réglementation à la culture de radioprotection*
}

\author{
M.C. BOEHLER**
}

\author{
(Manuscrit reçu le 6 septembre 1995, \\ révisé le 11 avril 1996, accepté le 18 avril 1996)
}

RÉSUMÉ Dans le souci de favoriser un haut degré de protection radiologique des travailleurs et des membres du public, les autorités compétentes et les exploitants de l'industrie nucléaire se doivent d'inciter les acteurs de la radioprotection à faire mieux que ne l'exigerait la mise en ouvre pure et simple de la réglementation relative à la limitation des doses. Le respect des normes et spécifications techniques est une condition nécessaire mais non suffisante pour une radioprotection de qualité. Atteindre cet objectif de qualité, ce n'est pas forcer les améliorations par une politique réglementaire de réduction des limites de doses, mais promouvoir une véritable culture de radioprotection fondée sur une démarche d'optimisation de la radioprotection formalisée au plus haut niveau de la hiérarchie de l'entreprise ainsi que sur le professionnalisme, la responsabilité personnelle, la motivation et l'engagement librement consenti et bien compris de tous les acteurs à mettre en ceuvre cette politique. La diffusion de la culture de radioprotection encourage l'adoption délibérée dans la pratique quotidienne d'un comportement de nature à diminuer aussi bas que raisonnablement possible les expositions aux rayonnements ionisants et à donner ainsi vie à "l'esprit" de la réglementation dans des conditions optimales. Cet article a pour objectif de montrer que la nécessité de la diffusion d'une culture de radioprotection, fondée notamment sur le principe d'optimisation, s'inspire de la philosophie même du système que recommande la Commission internationale de protection radiologique dans sa publication 60 pour la gestion du risque radiologique résiduel ainsi que de l'approche comportementale et incitative que suppose le principe d'optimisation. Un accent particulier sera porté sur les éléments fondamentaux susceptibles de contribuer à une définition de la culture de radioprotection.

ABSTRACT In order to encourage a high degree of radiation protection of workers and members of public, the relevant authorities and operators in the nuclear industry should urge those responsible for radiation protection to do more than is required by the pure and simple application of the regulations related to dose limitation. Compliance with the technical standards and specifications is a necessary but not sufficient condition for quality in radiation protection. Reaching this quality objective is not a matter of forcing improvements by a regulatory policy of reducing dose limits, but of promoting a real radiation protection culture based on an approach of optimizing the radiation protection formalised at the highest level of the company structure, as well as on the professionalism, individual responsability, motivation and the freely consented to and understood

\footnotetext{
* Communication présentée en anglais lors de la Conférence internationale "Radiation Dose Management in the Nuclear Industry" organisée par British Nuclear Energy Society, Windermere, Royaume-Uni, 9-11 octobre 1995.

** Électricité de France, Direction Générale/Service de Radioprotection, 3 rue de Messine, 75384 Paris Cedex 08, France.
} 
participation of those involved in implementing this policy. The spread of a radiation protection culture encourages the deliberate adoption in everyday practice of behaviour likely to reduce exposure to ionizing radiation, as low as reasonably achievable, and to give life to the "spirit" of the regulations in the best conditions. This article intends to show that the need to diffuse a radiation protection culture, particularly based on the principle of optimization, is inspired both by the philosophy behind the system recommended by the international Commission on radiological protection itself, in its publication 60 on the management of residual radiological risk, as well as by the behavioural and incentive approach implied by the optimization principle. Special attention will be given to the fundamentals likely to contribute in a definition of radiation protection culture.

\section{Introduction}

Il apparaît aujourd'hui nécessaire de promouvoir pour la protection radiologique une démarche dynamique fondée sur une approche comportementale qui ne soit plus uniquement fondée sur la stricte application réglementaire du principe de limitation des doses individuelles. Dans la logique de l'hypothèse qu'a retenue la Commission internationale de protection radiologique (CIPR) d'une relation linéaire et sans seuil considérée comme prudente au niveau des faibles doses, ce principe ne traduit pas un seuil "d'innocuité" ou a contrario un seuil "de danger". Il ne fait que permettre une distinction objective et quantifiable entre les niveaux de risque jugés inacceptables et ceux jugés tolérables. Il correspond à une limite "juridique" d'interdit qui se fonde tout autant sur des considérations sanitaires que sur des jugements économiques et sociaux.

Dans cette logique, il est nécessaire que l'on mette en œuvre, en complément de la radioprotection de contrôle a posteriori (optique du respect des limites de dose individuelles), une radioprotection de gestion a priori des doses individuelles et collectives fondée sur la fixation d'objectifs optimisés. L'application du principe d'optimisation ne supprime nullement la radioprotection traditionnelle de contrôle dont elle est tout à fait complémentaire.

La promotion de cette approche plus pragmatique de la protection radiologique résulte de l'évolution de la place et des fonctions des concepts de limite et d'optimisation de la protection radiologique consacrée par la publication 60 de la CIPR (1991). L'obligation de comportement à caractère incitatif qui soustend le principe d'optimisation motive les exploitants à faire diligence pour atteindre au mieux les objectifs dosimétriques qu'ils se sont fixés en matière de gestion des niveaux d'exposition résiduels tout en agissant au mieux des intérêts de la collectivité et de leurs intérêts propres dans le milieu concurrentiel où ils se trouvent placés.

Par delà le respect des procédures définies, les individus doivent agir conformément à une culture de radioprotection, entendue comme une approche de "gestion" des doses individuelles et collectives dans une optique 
d'application du principe d'optimisation. La protection radiologique doit s'appuyer sur l'adhésion et la participation motivée des différents acteurs à des objectifs optimisés de réduction des doses. C'est cette nouvelle étape du développement de la culture de radioprotection qui doit être franchie dans la même logique que celle qui est poursuivie dans le domaine de la sûreté. La diffusion d'une culture de radioprotection fondée notamment sur le principe d'optimisation est indispensable pour faire de la radioprotection un élément à part entière dans le management des installations nucléaires.

\section{La place du principe de limitation des doses}

Dans la publication 26 de la CIPR (1977) et plus récemment dans la publication 60 , on peut constater une évolution importante quant à la hiérarchisation des principes fondamentaux de la protection radiologique qui sont la justification de la pratique, l'optimisation de la protection radiologique (généralement repris sous l'acronyme ALARA - As Low As Reasonably Achievable - dans la terminologie de la CIPR) et la limitation des doses individuelles. Le système qu'elle propose ne s'appuie plus exclusivement, comme auparavant, sur des doses maximales admissibles utilisées comme limite supérieure du risque acceptable. Le concept de limite, dans sa dimension "juridique" d'interdit, ne constitue plus l'assurance d'une protection radiologique de qualité.

Le système de prévention fondé sur la notion de seuil a cédé la place au système de gestion du risque radiologique fondé sur le principe de précaution lié à la reconnaissance des effets stochastiques dès 1950 et à l'adoption ultérieure de l'hypothèse d'une relation linéaire et sans seuil considérée comme prudente au niveau des faibles doses. L'adoption de cette hypothèse, qui n'est pas la traduction d'une connaissance scientifique mais une construction intellectuelle destinée à fonder l'action en matière de radioprotection, a impliqué un certain nombre de conséquences sur la place et les fonctions des concepts de limite et d'optimisation dans le système de protection radiologique. La limite est maintenant considérée comme la frontière basse de la région des doses inacceptables. Les valeurs au dessus de la limite doivent être très strictement réglementées, et les doses en dessous de la limite ne sont considérées comme acceptables que dans la mesure où les niveaux d'exposition résiduels sont optimisés.

La limitation des doses ne doit donc plus être envisagée ni comme la finalité de la radioprotection ni être posée en seul et unique postulat du système de protection radiologique. Dans cette optique, la CIPR, au paragraphe 124 de sa publication 60, rappelle que dans la pratique un certain nombre d'idées fausses sont apparues à propos de la définition et de la fonction des limites de doses. Ces dernières sont généralement, et de façon erronée, appréhendées d'une part, comme la frontière entre ce qui est sûr et ce qui est dangereux et, d'autre part, comme le moyen le plus simple et le plus efficace de maintenir à des 
niveaux suffisamment bas les expositions et de forcer les améliorations, étant entendu. que ces limites sont souvent considérées comme la seule mesure de rigueur dans un système de protection.

Ces idées fausses sont, dans une certaine mesure, renforcées par le fait que ces limites sont transposées dans les réglementations. Il est vrai que, alors que l'effort doctrinal de la CIPR porte sur le principe d'optimisation, c'est paradoxalement la fixation des limites qui demeure la principale préoccupation de ceux qui ont la charge de transcrire en normes juridiques le système de gestion du risque radiologique recommandé par la CIPR. En fait, une réglementation contraignante de la protection radiologique fondée sur la notion de seuil juridique établie sur les doses maximales admissibles semble garder la faveur des pouvoirs publics qui fonctionnent essentiellement dans une approche de type "juridique et réglementaire". Le dépassement des limites devient alors une infraction à la réglementation. Dans ce contexte, il n'èst pas étonnant que les autorités compétentes préfèrent fonder leur action de contrôle sur le respect des limites de doses, même quand les sources sont partiellement ou même entièrement hors de leur contrôle, alors que l'optimisation de la protection ápparaît comme la ligne de conduite la plus appropriée.

\section{Le rôle fondamental du principe d'optimisation}

La réglementation en radioprotection doit avoir deux vertus. Elle doit non seulement être contraignante par le respect des limites de doses dans le contexte de la radioprotection de contrôle a posteriori. Mais elle doit aussi et surtout être incitative par la promotion de l'optimisation et l'amélioration des comportements dans le contexte de la radioprotection de gestion a priori des doses individuelles et collectives fondées sur la fixation d'objectifs dosimétriques optimisés. Toute démarche visant à motiver les différents acteurs de la radioprotection doit en premier lieu restituer le principe d'optimisation dans son contexte réglementaire. Le respect de ce principe est tout aussi primordial que celui de la limite de dose individuelle, conformément à la séquence logique que traduit par exemple la réglementation française dans la transposition des principes fondamentaux de la, protection radiologique : "Les matériẹls, les procédés et l'organisation du travail doivent être conçus de telle sorte que les expositions professionnelles individuelles et collectives soient maintenues aussi bas que raisonnablement possible en dessous des limites prescrites" (article $8 \S 2$ du décret du 28 avril 1975 relatif à la protection des travailleurs dans les installations nucléaires de base, modifié par le décret du 6 mai 1988 (Décrets 75-306 et 88-662) - article $4 \cdot$ du décret du 2 octobre 1986 relatif à la protection des travailleurs hors des installations nucléaires de base, modifié par le décret du 6 mai 1988 (Décrets 86-:103 et 88-662)).

Le rôle fondamental accordé au principe d'optimisation, consacré par la publication 60 de là CIPR, montre que ce principe, que l'on pourrait désormais qualifier de "clé de voûte" du système de protection radiologique, est le garant, 
non plus seulement d'une bonne protection dont on se "contentait" lorsqu'on se conformait au principe de limitation des doses, mais d'une nouvelle logique de protection rationnelle et efficace fondée sụr la recherche d'un arbitrage entre les coûts de protection et les niveaux d'exposition résiduels individuels et collectifs. L'optimisation de la protection, par ses considérations d'ordre économique, politique et social mais aussi sanitaire, oriente davantage l'action réglementaire vers l'édification d'un consensus social sur les choix technologiques impliquant l'utilisation des rayonnements ionisants.

En privilégiant une dynamique de réduction des doses ne visant pas à approcher le risque nul, l'énoncé du principe d'optimisation constitue à bien des égards une innovation importante par rapport à l'état général des pratiques en matière de normes de sécurité et de protection. İl est en effet probable que ce soit la première fois dans ces domaines que l'on consente à aller au delà d'une conception de type juridique des normes matérialisées dans une donnée quantitative exprimant une certaine limite à ne pas dépasser.

L'énoncé d'une valeur limite peut alors apparaître comme un pis-aller plutôt que comme une solution idéale, dès lors que l'on a admis par prudence les effets potentiels des faibles doses. Non seulement elle n'intègre pas dans sa logique une incitation à réduire les niveaux d'exposition en deçà du niveau maximum requis par la législation, même si cette réduction apparaît techniquement et économiquement envisageable, mais elle peut même aussi apparaître comme une solution susceptible d'encourager implicitement les exploitants à se situer au voisinage de ce niveau maximum imposé par la législation. C'est le concept d'optimisation qui motive les exploitants à faire un effort de réduction des doses tout en assurant une allocation optimale de leurs ressources.

Le principe de limitation des doses joue désormais le rôle d'une garantie individuelle qui agit comme correctif ou butoir au libre jeu de l'optimisation. Il apporte un éclairage particulier aux principes de justification et d'optimisation qui, pris séparément ou ensemble, pourraient laisser craindre que des considérations économiques et sociales mal comprises puissent induire des choix erronés, voire dangereux. Dans cette logique, on peut considérer que l'approche de l'optimisation est "protectrice" sur les plans individuel et collectif dans la mesure où elle permet une protection optimale pour l'ensemble des individus, la limitation jouant un rôle de garantie individuelle uniquement dans le cas où certains de ces individus subiraient des doses excessives. C'est pourquioi la publication 60 de la CIPR, réaffirmant le rôle primordial de l'optimisation, précise que les doses et les risques doivent être optimisés dans le cadre des limites de dose et de risque spécifiés pour. les individus.

De plus, les dernières recommandations de la CIPR reflètent une évolution conceptuelle intéressante dans la mesure où elles mettent l'accent sur l'une des finalités éthiques du principe d'optimisation, à savoir la recherche de l'équité dans la distribution des doses individuelles. La prise en compte de l'équité apparaît comme une évolution importante dans le système de protection radio- 
logique. Il est en effet reconnu explicitement que les situations auxquelles les personnes sont exposées mais également les actions en matière de protection peuvent être génératrices d'inégalités d'exposition jugées suffisamment importantes pour devoir être atténuées. L'objectif de l'optimisation n'est donc plus seulement de réduire les expositions aussi bas qu'il est raisonnablement possible en tenant compte des considérations économiques et sociales mais aussi de veiller en priorité à réduire les expositions des personnes ayant les niveaux de dose individuels les plus élevés. La notion de contrainte de dose répond notamment à cette préoccupation. Ce concept que l'on peut définir comme une restriction quantifiée sur les doses individuelles introduite pour la mise en œuvre du processus d'optimisation afin de "réduire l'iniquité" dans la distribution des expositions individuelles associées à un type de source donnée soulève quelques interrogations liées d'une part, à son articulation par rapport au principe d'optimisation auquel il est associé et que l'on définit comme la réduction des expositions aussi bas que raisonnablement possible compte tenu des facteurs économiques et sociaux - donc non quantifié a priori - et, d'autre part, au risque de confusion qu'il pourrait engendrer avec le principe de limitation des doses.

Dans sa publication 60, la CIPR consacre l'évolution relative à la place et aux fonctions des concepts de limite et d'optimisation en clarifiant conceptuellement le modèle d'acceptabilité du risque radiologique. La notion de limite se fonde désormais sur le concept de tolérabilité du risque. La limite d'exposition est définie comme la frontière entre ce qui est "inacceptable" et ce qui est "tolérable". Dans l'optique de la CIPR, le terme "inacceptable" est utilisé pour indiquer qu'en situation normale, tout doit être mis en œuvre en deçà de la frontière pour réduire les expositions vers le champ du risque résiduel "tolérable". Quant au "tolérable", il convient d'introduire une distinction supplémentaire entre les situations qui ne sont pas réellement satisfaisantes mais considérées néanmoins "tolérables" et celles qui sont non seulement "tolérables" mais également "acceptables" lorsque la protection est optimisée. Ainsi, il est possible de considérer que le risque résiduel "tolérable" devient acceptable dès lors que la protection est optimisée, ainsi que le montre la figure 1.

\section{La nature juridique du principe d'optimisation}

Les techniques juridiques traditionnelles du droit administratif "classique" qui se fondent sur la notion de seuil et qui sont adaptées à la transposition juridique du principe de limitation des doses individuelles ne répondent cependant pas exactement aux problèmes que pose la mise en œuvre du principe d'optimisation. Inspirée d'une attitude de précaution, la démarche que suppose le principe d'optimisation, qui correspond à la gestion du risque envisagée comme la recherche de l'acceptable à un moment donné dans un contexte donné, se traduit pour l'essentiel par le recours à des techniques plus souples d'incitation et de concertation (Lochard et Boehler, 1993). 


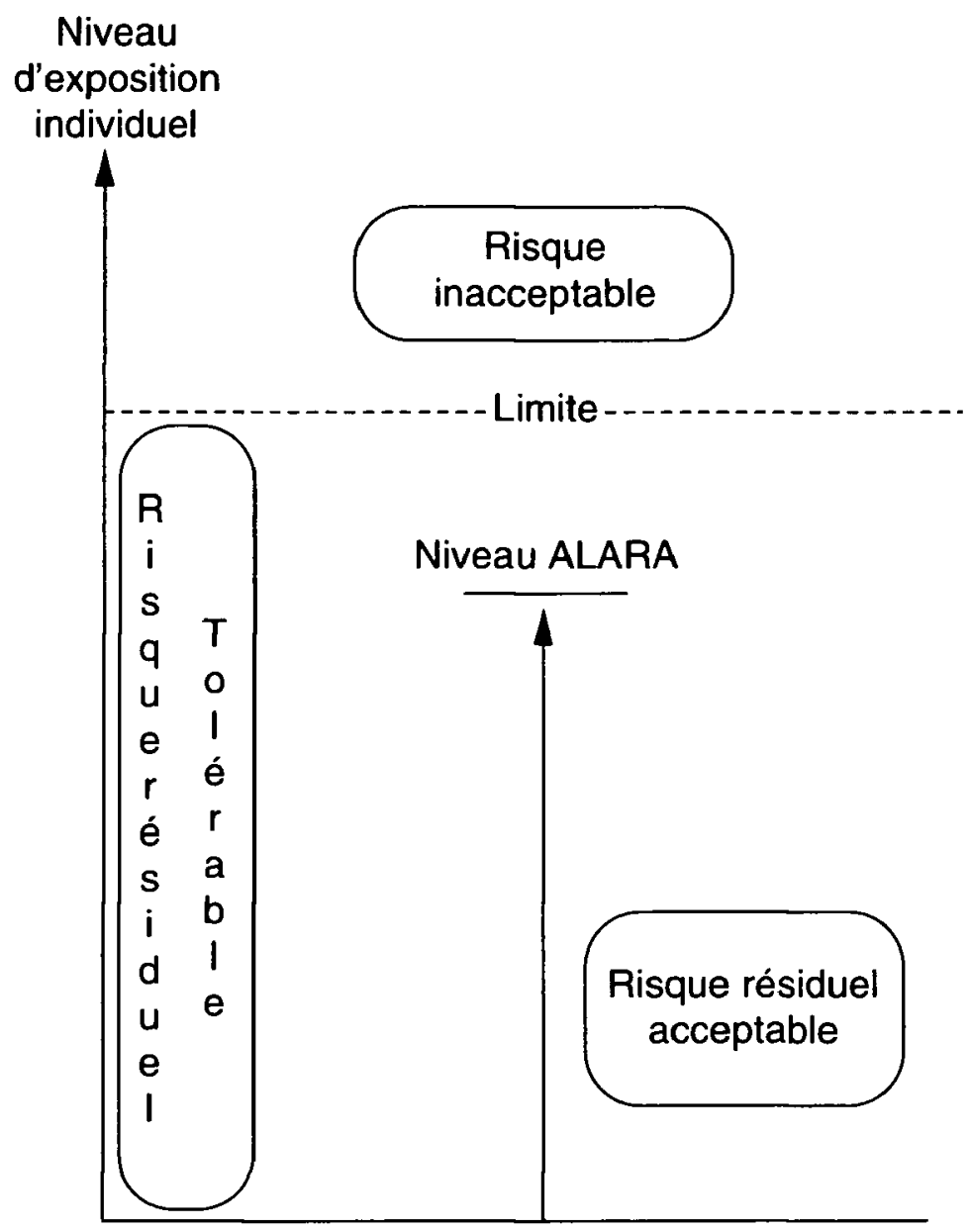

Fig. 1. - Le modèle de la tolérabilité du risque résiduel. Model of tolerability of residual risk.

L'optimisation implique en effet que, par une analyse détaillée d'opérations envisagées et partant d'une expérience dans des situations similaires, on recherche les voies d'amélioration possibles et l'on décide, le cas échéant, la mise en œuvre de moyens supplémentaires à un coût jugé acceptable. L'exploitant définit ainsi des objectifs de dose à atteindre. Afin d'atteindre au mieux les objectifs dosimétriques qu'il s'est fixés, l'exploitant s'appuie dans sa démarche sur une obligation de comportement des individus, laquelle requiert de sa part un effort de formation, de sensibilisation, de responsabilisation des travailleurs qu'il peut éventuellement être amené à justifier. Compte tenu du caractère incitatif de la démarche, dans une recherche d'une meilleure qualité, le fait de ne pas atteindre les objectifs ne peut pas en soi être sanctionnable. Les objectifs chiffrés que s'assigne l'exploitant pourraient servir de point de référence pour. un contrôle, par les pouvoirs publics, de l'application du principe d'optimisation. Ceci ne signifie pas pour autant qu'il faille en déduire qu'une non atteinte des objectifs internes de l'exploitant constitue une infraction. 
Dans la mesure où la dynamique de l'optimisation est essentiellement à caractère incitatif et qualitatif, une réglementation ne peut faire intervenir l'optimisation que comme une exigence générale, de la souplesse étant requise dans son application au plan réglementaire, complétée par le recours à des documents d'orientation. La communication 85-C347-03 du 31 décembre 1985 de la Commission de l'Union européenne sur la mise en cuvre des directives du Conseil 80-836-Euratom du 15 juillet 1980 et 84-467-Euratom du 3 septembre 1984 le suggère d'ailleurs de façon claire quand elle énonce que "les principes fondamentaux de justification et d'optimisation des expositions n'ont à l'évidence qu'une valeur générale, ce dont il y a lieu de tenir compte lors de leur transposition dans les dispositions législatives, réglementaires et administratives nationales". Elle ajoute que "le troisième principe (limitation des doses) peut, quant à lui, être transposé sans restriction dans une législation nationale à caractère contraignant".

L'application du principe d'optimisation peut être assimilée à la mise en œuvre de "règles de l'art" qui apparaissent comme des normes de comportement qu'il s'agisse de comportement d'ordre intellectuel ou d'ordre matériel. Elles sont constituées par un appel à la recherche de modalités d'action inspirées de la prudence et de la diligence. Le principe d'optimisation s'apparente à un savoir faire de qualité et doit être compris comme un moyen d'inciter les exploitants à une vigilance optimale consistant à tout mettre en œuvre pour réduire les expositions professionnelles aussi bas que raisonnablement possible compte tenu des facteurs économiques et sociaux.

L'effort constant de l'exploitant en vue de mettre en œuvre l'objectif d'optimisation est ici l'objet de l'obligation. L'exploitant peut donc être tenu pour responsable non pas de ne pas avoir atteint un résultat précis et chiffré correspondant à une dose optimisée, mais de ne pas avoir mis en œuvre les actions raisonnables pour réduire les doses. On peut imaginer toutes les difficultés d'interprétation susceptibles de survenir en matière d'appréciation soit par l'autorité compétente, soit par le juge, du caractère supposé raisonnable ou non de ces actions. Il est en effet extrêmement difficile de déterminer si l'optimisation a été atteinte dans la mesure où le but, considéré comme un objectif de qualité plutôt que comme un objectif de dose spécifique, ne peut que recevoir une définition variable en fonction de la spécificité des situations particulières (différente allocation des ressources, évolution des technologies disponibles...).

Dans la pratique, il serait souhaitable que l'exploitant n'encoure la charge de la responsabilité que si son comportement est manifestement fautif, la faute pouvant être une erreur de conduite quand il y a contradiction évidente entre les actes et le comportement raisonnable attendu, une négligence ou une erreur d'appréciation graves susceptibles ou ayant eu des conséquences non négligeables sur le plan de la protection radiologique. C'est un champ cependant plus "large" de responsabilité de l'exploitant qui a été retenu dans une affaire où British Nuclear Fuels (BNFL) a été condamné en 1985 pour un manquement au principe d'optimisation dans le cas d'un rejet d'effluents radioactifs 
dans la Mer d'Irlande en 1983. Le motif d'accusation ne portait pas sur le fait que des expositions significatives de personnes extérieures au site étaient survenues. L'argument était plutôt de faire prévaloir qu'il n'était pas nécessaire qu'une telle quantité d'effluents radioactifs soit rejetée en mer et qu'elle aurait pu être évitée par l'exercice d'une action raisonnable de la part de BNFL. En conséquence, le principe d'optimisation a été considéré comme non respecté.

Il faut noter que le droit connaît depuis longtemps l'obligation de comportement avec la gestion en bon père de famille (bonus paterfamilias) qui correspond à l'obligation imposée à un personnage standard : l'homme raisonnable. Il semble bien que l'obligation de l'exploitant, dans le cas de l'optimisation, lui impose la conduite du "bon père de famille"...

\section{La mise en ouvre de la culture de radioprotection}

Comme il ne peut y avoir d'industrie du nucléaire sans culture de sûreté de chacun des acteurs, il ne peut y avoir de pérennité et de réelle acceptation par l'opinion publique de la production d'électricité d'origine nucléaire sans culture de radioprotection de ces mêmes acteurs, depuis les pouvoirs publics jusqu'à l'intervenant sur le chantier (Boehler, 1994). La phase de prise en compte purement technique de la radioprotection (intégration de la radioprotection dans la conception des installations, prise en compte de la radioprotection dans les procédures et modes opératoires...) est fondamentale mais insuffisante. Il apparaît nécessaire que d'une part, elle soit accompagnée d'une démarche organisationnelle englobant la préparation, le suivi des chantiers, le retour d'expérience selon notamment les méthodes ALARA et que d'autre part, soit franchie une nouvelle étape reconnaissant au facteur humain une place prépondérante dans la qualité de l'exploitation qui se traduise dans la pratique par le développement de la culture de radioprotection.

La recherche du "mieux" dans un effort permanent d'amélioration de la qualité des actions se retrouve derrière la formule "culture de radioprotection" qui est essentiellement fondée sur :

- l'acceptation par les individus, dans le doute, qu'un risque radiologique "potentiel" aux faibles doses existe et que la protection radiologique a pour but de préserver leur santé ;

- les connaissances et les compétences en matière de protection radiologique, conférées par la formation théorique et pratique, par une bonne circulation de l'information et par une large et rapide diffusion du retour d'expérience national et international ;

- l'engagement, à savoir l'implication de la hiérarchie dans une politique de radioprotection, et l'adhésion des individus à l'objectif commun de réduction des expositions aussi bas que raisonnablement possible ;

- la motivation, par le biais de la fixation d'objectifs et grâce à l'effort de responsabilité personnelle et d'autodiscipline de groupe en matière de radioprotection ; 
- la supervision et le contrôle, comprenant des pratiques d'audit;

- la responsabilité, notamment par une attribution claire des missions respectives des intervenants tant de l'exploitant que des entreprises extérieures.

Si l'on s'inspire de la définition qui est donnée de la culture de sûreté dans le document INSAG 4 du Groupe consultatif international pour la sûreté nucléaire publié par l'Agence internationale de l'énergie atomique, on pourrait définir de la façon suivante la culture de radioprotection : "la culture de radioprotection représente l'état d'esprit par rapport à la radioprotection, à savoir l'image que chaque individu ou chaque organisation s'en fait, la valeur qui lui est donnée et l'intérêt qui lui est porté. Cet état d'esprit conditionne les attitudes èt pratiques mises en auvre dans l'activité professionnelle". La diffusion de la culture de radioprotection doit correspondre dans la pratique à une dynamique de remise en question quand il le faut des modes de pensée et de travail pour que soit dévolue à la radioprotection la place qui doit lui être accordée en fonction de son importance. La définition susmentionnée met en exergue le fait que la culture de radioprotection est tout autant une question d'attitude que de structure qui concerne à la fois les individus et les organismes et qui exige l'engagement permanent des responsables comme des exécutants tant au niveau de la conception que de l'exploitation des installations.

\subsection{Engagement de la hiérarchie et intégration dans le management}

Un engagement explicite de la hiérarchie de l'exploitant apparaît comme un facteur clé dans la diffusion de la culture de radioprotection. Cet engagement ne peut exister et perdurer que si la direction veut une culture de radioprotection parce qu'elle estime que tel est l'intérêt de l'entreprise pour deux raisons majeures. D'une part, parce qu'elle est arrivée à la conclusion que le coût à prévoir pour réaliser des performances dans le domaine de la protection radiologique ne saurait compromettre l'atteinte des objectifs prioritaires de l'entreprise. Le principe d'optimisation étant un point de repère, une référence pour l'entreprise lui rappelant la nécessité de réaliser la meilleure allocation possible des ressources de protection et d'éviter donc les gaspillages, il s'inscrit dans la logique même du management de l'entreprise. Le principe d'optimisation intervient pour corriger les coûts de protection déraisonnables et "rentabilise" les efforts consentis pour une réduction "rationnelle" des expositions. D'autre part, parce qu'elle est persuadée que la diffusion d'une telle culture fondée sur une politique de réduction des doses peut participer d'une amélioration de son "image" auprès du public ainsi qu'auprès de son personnel.

La formulation de la volonté de diffuser cette culture doit être suffisamment claire pour que le personnel soit convaincu que ses performances dans le domaine de la protection radiologique seront un critère d'appréciation important, au même titre que les performances industrielles et commerciales. Afin qu'une véritable culture de radioprotection fondée en particulier sur le principe d'optimisation soit totalement intégrée au management de l'entreprise, il est fondamental que la direction impulse le mouvement par une déclaration de 
politique générale et qu'elle démontre ensuite par toute son attitude qu'elle s'engage pleinement vis-à-vis de son personnel à agir de façon à promouvoir cette culture dans l'entreprise. Dans la pratique, elle doit adopter une attitude concrète et effective consistant à veiller à ce que les processus qui influent sur la radioprotection soient examinés de façon régulière et à s'intéresser directement aux questions les plus importantes touchant à la radioprotection, tout en rappelant le message de fond qui est que si l'on veut être exemplaire en matière dosimétrique, il faut mettre en œuvre le principe d'optimisation (CEPN, 1994).

À EDF, l'engagement de la hiérarchie au plus haut niveau s'est essentiellement traduit d'une part, par la publication en juin 1993 du Livre blanc de la radioprotection qui traite des orientations et des objectifs de la radioprotection à EDF, chaque objectif faisant l'objet d'un plan d'actions devant aboutir d'ici l'an 2000 et, d'autre part, par la désignation d'un membre du Comité de direction chargé de la mise en œuvre de l'optimisation. En décembre 1991, EDF s'est fixée comme objectif de mieux intégrer la radioprotection ALARA pour le personnel dans le management global de l'entreprise en faisant passer l'objectif de réduction optimale des expositions au rang d'objectif prioritaire au même titre que la disponibilité des tranches (Stricker et Dollo, 1995). La dose collective moyenne par tranche était effectivement passée progressivement de 1,77 H Sv en 1988 à 2,44 H Sv en 1991, après une période de dix ans où les résultats obtenus en France étaient meilleurs que ceux des autres grands pays nucléaires (Etats-Unis, Japon, Allemagne). Le programme d'action s'appuyant sur une démarche ALARA, lancé en 1992 par le parc nucléaire d'EDF, a permis d'inverser la tendance : on a enregistré en 1992 une dose moyenne par réacteur de 2,36 H Sv (et ce malgré l'incidence des interventions sur les couvercles de cuve $-7 \%$ -), qui a été diminuée en 1993 à 2,04 H Sv, et à 1,74 H Sv en 1994. Un objectif dosimétrique global dans le plan stratégique du parc de $1,6 \mathrm{H} \mathrm{Sv}$ /réacteur.an en moyenne à l'échéance de 1995 a été défini : la dose collective moyenne a été ramenée à 1,63 H Sv en 1995. On peut donc considérer que l'objectif fixé à 1,6 H Sv pour 1995 a été atteint. La fixation de cet objectif global a été relayée par une politique de négociation puis d'intégration d'objectifs dosimétriques par site, tranche, arrêt et opération dans les contrats de gestion annuels et les plans stratégiques trisannuels sur lesquels les chefs de sites s'engagent vis-à-vis de la Direction nationale. Le parc nucléaire, à partir de la programmation pluriannuelle des travaux et de l'analyse du retour d'expérience, s'est fixé l'objectif de 1,2 H Sv/réacteur an en moyenne à l'échéance de l'an 2000.

\subsection{Définitions des responsabilités}

L'autorité compétente en matière de radioprotection à l'échelon national doit pouvoir contraindre l'entreprise à diffuser une culture de radioprotection notamment en tenant la hiérarchie de l'entreprise comme responsable en particulier de la mise en cuvre des moyens nécessaires au respect du principe d'optimisation. En France, la réglementation précise que : "l'exploitant d'une ou plusieurs installations nucléaires de base a la responsabilité de toutes les 
mesures générales d'ordre administratif et technique, notamment en matière d'organisation du travail, nécessaires pour la prévention des accidents du travail et des maladies professionnelles susceptibles d'être causés par les rayonnements ionisants". (article 2 du décret du 28 avril 1975). Cette disposition juridique mettant l'organisation du travail à la charge de l'exploitant qui, de surcroît, peut seul agir dans la pratique sur les débits de dose, fait de celui-ci le débiteur principal de l'obligation d'optimisation de la radioprotection dans les installations nucléaires. A EDF, la responsabilité de la mise en œuvre de l'optimisation serait vraisemblablement imputée à la personne du chef de centrale nucléaire en tant que représentant de l'exploitant sur le site.

Le rappel des responsabilités sur le plan juridique de la hiérarchie de l'entreprise doit s'accompagner à la fois d'une allocation des ressources et des structures nécessaires à la diffușion de la culture de radioprotection à tous les niveaux de l'entreprise ainsi que d'une délégation de responsabilités dans la pratique complétée par une définition claire des tâches et des moyens correspondants. Pour que l'environnement de travail du personnel favorise la mise en cuvre de la culture de radioprotection, il est nécessaire que les moyens financiers soient suffisants pour que les agents devant s'acquitter des tâches liées à la radioprotection aient à leur disposition le matériel, les installations et l'infrastructure technique nécessaires. Une définition correcte des tâches des acteurs de la radioprotection est indispensable de façon à éviter toute ambiguïté et tout problème de responsabilités partagées qui pourraient dans la pratique avoir pour conséquence des chevauchements, des omissions ou une dilution des responsabilités. Cette exigence de rigueur dans la définition des tâches de chacun et la délégation de pouvoirs correspondante notamment dans le domaine de l'organisation du travail participe de la nécessité d'identifier les fonctions (et donc les personnes) susceptibles d'être, directement ou indirectement à l'origine d'une défaillance dans le système de protection radiologique. Cette définition des responsabilités doit être perçue comme étant avant tout une volonté de prévention destinée à identifier les erreurs commises pour en éviter le renouvellement, et non comme une volonté de sanction fondée sur la recherche des manquements à l'origine de ces erreurs.

\subsection{Attitudes du personnel}

Si: l'impulsion hiérarchique est nécessaire pour le succès de la mise en œuvre d'une véritable culture de radioprotection et en particulier de l'optimisation, elle n'est pas suffisante. Une des conditions primordiales pour la réussite de tout système de gestion des doses est l'engagement de l'enșemble du personnel à tous les niveaux hiérarchiques de l'entreprise. La véritable clé du succès repose en effet sur la motivation des intervenants eux mêmes et donc dans leur sensibilisation à leur responsabilité personnelle et collective en matière de radioprotection. L'objectif doit être de faire accepter par tous que le principe d'optimisation est une démarche de progrès qui apporte souvent des améliorations qui dépassent le cadre strict de la radioprotection, plutôt qu'une contrainte supplémentaire décidée par la hiérarchie: La motivation de tous les 
acteurs est primordiale dans la phasè de préparation et pendant les opérations afin' de réduire les dysfonctionnements qui peuvent occasionner dans la pratique en France jusqu'à $30 \%$ de la dose. Leur coopération doit aller au-delà d'un engagement ponctuel pour une opération donnée, et justifie un effort permanent et continu car-le retour d'expérience montre que rien n'est jamais acquis en matière de radioprotection.

Pour que le principe d'optimisation devienne un véritable critère dynamique de responsabilité professionnelle dans les activités nucléaires, les intervenants doivent systématiquement adopter une démarche rigoureuse et prudente fondée sur le professionnalisme et le "temps de la réflexion". en. privilégiant une attitude interrogative, et pallier entre eux tout déficit de communication. Le développement du professionnalisme suppose la mise en œuvre d'une politique de formation ALARA et d'intégration de l'esprit ALARA dans l'entraînement du personnel, puis ultérieurement des recyclages périodiques. La mobilisation du personnel pendant les opérations passe par une information permanente sur les objectifs et les résultats ainsi que par une politique d'inçitation qui consiste par exemple à prendre en compte dans l'évaluation individuelle annuelle l'engagement par rapport à là démarche ALARA. La stimulation d'une attitude interrogative peut être concrétisée par des "check lists" ALARA utilisées lors des revues de projet ante et post chantier, correspondant davantage à une incitation à la réflexion qu'à la formulation de prescriptions.

En vue de développer la culture de radioprotection de l'ensemble des acteurs de l'électronucléaire et notamment les entreprises sous-traitantes, EDF s'est engagée sur la voie d'un vaste programme de sensibilisation et de formation ALARA qui vise en priorité les acteurs des arrêts de tranche, et qui a pour objet d'intégrer ALARA dans la formation aux métiers. Un module ALARA a ainsi été inclus dans les habilitations en radioprotection nécessaires pour travailler en zone contrôlée, et des modules de même type doivent être systématiquement intégrés dans les formations aux métiers, y compris pour ceux qui ne travaillent pas en zone mais dont les tâches interagissent sur les expositions professionnelles. Pour motiver tant son personnel que celui des entreprises extérieures, EDF s'efforce de porter actuellement ses efforts sur l'amélioration de la circulation de l'information et de la diffusion rapide du retour d'expérience national et international qui participent fondamentalement de la réussite de toute politique ALARA. Un effort particulier a été entrepris par EDF pour favoriser des politiques de communication et de sensibilisation adaptées à chaque site et qui fassent appel à l'imagination et à la créativité des agents.

\section{Conclusion}

L'introduction de la culture de radioprotection fondée sur le principe d'optimisation dans le management de l'entreprise modifie la philosophie de l'action et les comportements. A tant parler aujourd'hui d'éthique en radioprotection, les experts ne sacrifient pas à un phénomène de mode mais à une cer- 
taine prise de conscience du fait que la réflexion sur le risque radiologique est au moins autant de nature comportementale que technicienne. Le développement de la culture de radioprotection fondée sur le principe d'optimisation répond à ces préoccupations éthiques par le comportement raisonnable fondé sur des jugements de valeur d'ordre social, économique et de protection sanitaire et par la responsabilité dans l'action qu'elle suppose. La réussite d'une gestion optimisée des expositions, basée sur la motivation de tous les acteurs concernés, s'appuie sur une politique d'information et de transparence en ce qui concerne les risques résiduels dus aux rayonnements ionisants et les moyens mis en œuvre pour les réduire aussi bas que raisonnablement possible. La diffusion et l'excellence pratique de la culture de radioprotection seront probablement déterminantes dans une dynamique d'amélioration de la communication et de l'acceptation par le public du risque radiologique.

\section{RÉFÉRENCES}

Boehler M.C. (1994) Culture Radioprotection - Amélioration de la culture de radioprotection et comportement ALARA, Rapport n ${ }^{\circ} 224$ du Centre d'étude sur l'Evaluation de la Protection dans le domaine Nucléaire, Fontenay-aux-Roses, décembre 1994.

Centre d'étude sur l'Evaluation de la Protection dans le domaine Nucléaire (1994) L'optimisation de la radioprotection des travailleurs. In : Journées SFRP, "L'optimisation de la radioprotection des travailleurs dans les domaines électronucléaire, industriel et médical", La Rochelle, 20 et 21 septembre 1994, Rapport CEPN n 233, novembre 1994.

Communication 85-C347-03, JOCE C347-9 du 31/12/1985.

Décret $n^{\circ} 75-306$ du 28 avril 1975 relatif à la protection des travailleurs contre les rayonnements ionisants dans les installations nucléaires de base, JORF du 30/04/1975, modifié par le décret $n^{\circ} 88-662$ du 6 mai 1988, JORF du 08/05/1988.

Décret $n^{\circ} 86-1103$ du 2 octobre 1986 relatif à la protection des travailleurs contre les rayonnements ionisants hors des installations nucléaires de base, JORF du 12/10/1986, modifié par le décret n ${ }^{\circ} 88-662$ du 6 mai 1988, JORF du 08/05/1988.

Directive du Conseil 80-836-EURATOM du 15 juillet 1980 portant modification des directives fixant les Normes de base relatives à la protection sanitaire de la population et des travailleurs contre les dangers résultant des rayonnements ionisants, JOCE L.246$1 \mathrm{du} 17 / 09 / 1980$.

Directive du Conseil 84-467-EURATOM du 3 septembre 1984 modifiant la directive 80836-EURATOM en ce qui concerne les Normes de base relatives à la protection sanitaire de la population et des travailleurs contre les dangers résultant des rayonnements ionisants, JOCE L.265-4 du 05/10/1984.

Électricité de France (1993) Comité de Radioprotection, Groupe de Coordination en Radioprotection, Livre Blanc de la Radioprotection - La radioprotection. à EDF, Orientation et objectifs, Paris, juin 1993.

International Commission on Radiation Protection, (1977) ICRP publication 26, Pergamon Press, Oxford.

International Commission on Radiation Protection (1991) ICRP publication 60, Pergamon Press, Oxford.

Lochard J., Boehler M.C. (1993) Les bases éthiques et juridiques du principe d'optimisation de la radioprotection, Bulletin de Droit Nucléaire $n^{\circ} 52$, décembre 1993 , AEN/OCDE, pp. 9-28.

Stricker L., Dollo R. (1995) La politique ALARA d'Electricité de France, Radioprotection 30, 47-60. 